\title{
DOI 10.22448/AMJ.2017.3.22-22 \\ THE DEVELOPMENT OF MODERN PHARMACY EDUCATION IN OUR COUNTRY
}

\author{
Cheng Wei, Wang Haiyan, Wang Ying
}

Harbin Commercial University, 1 Xue Hai Street, Song Bei District, Harbin, 150028, chw@hrbcu.edu.cn

Hei Longjiang University of Chinese Medicine, 24 He Ping Road, Xiang Fang District, Harbin, 150040, xiaohanyan@126.com, wyviolin85@163.com

Objective: Chinese medicine and pharmacy education have not well defined since ancient times. In 1906, army medical school set up medicine and pharmacy education, which represents the beginning of Chinese modern pharmacy education. Looking back upon the development of pharmacy education, its achievement and lessons, we can get experience and guidance for future development of pharmacy education.

Method: Literature review, inductive and summary method. This paper studies Chinese pharmacy education in modern history stage. This paper also tries to outline its development history and features by doing review and research on related materials. And it compares the development process of Chinese and western pharmacy education.

Conclusion: The introduction of western culture had a great impact on traditional Chinese medicine and pharmacy system. Modern pharmacy education boomed, while its primary, secondary and higher school institutions still need to develop. Its establishment provided the basis for pharmacy education and trained talents for Chinese medicine and pharmacy.

\section{References}

[1] Zhao Lixun, et al. The Compendium of Works of Past and Present, Yi Bu Xu Lu[M]. Beijing: Chinese Science and Technology Publishing House, 2003:608

[2] Zhen Zhiya, et al. Chinese Medical History[M]. Shang Hai: Shanghai Science and Technology Publishing House, 1984:52-80, $112-116$

[3] Mu Jingqiang, Western Medicine History : the Localization of Western Medicine Education in the Public of China[M]. Beijing: Chinese Union Medical University Press. 2010

[4] Chen Zhijin. The Development of Pharmacologic Education in New China[J]. Chinese Pharmaceutical Bulletin, 1957,5(9): 347

[5] Song Wusheng. What is Pharmacy and General Situation of Pharmacologic Education in China[J]. Chinese Journal of Materia Medica, 1940, 2(2): 44

[6] Xue Yu, et al. Chinese Pharmaceutical History[M]. Beijing: People's medical Publishing House, 1984:356-384

[7] Li Tao. Medical Education in the 21 years of Republic of China[J]. Chinese Medical Journal, 1933, 19(5): 681

[8] Yang Ziyuan. The Brief Introduction of Shenyang Pharmacy College[J]. Chinese Pharmaceutical Bulletin, 1980,15(4):162

[9] Department of Pharmacy of Shanghai First Medical College. The Brief Introduction of Department of Pharmacy of Shanghai First Medical College[J]. Chinese Pharmaceutical Bulletin,1980,15(8):359

[10] The Brief Introduction of Chinese Universities[M]. Beijing: Science and Education Press. 1982:324,401,566,301,250,33

[11]Jiang Huiming. Retrospective and Prospective of Chinese Medical Education[J].Chinese Western Medicine.1935,8(1):50

[12]Xiao Zhuoyin. The Brief Introduction of Department of Pharmacy in Si Chuan Medical College[J]. Chinese Pharmaceutical Bulletin.1980,15(6):274

[13] The Academic Affairs of Nanjing Pharmaceutical College. The Brief Introduction of Nan Jing Pharmaceutical College[J]. Chinese Pharmaceutical Bulletin.1980,15(6):274

[14]Si Yuanyi. Higher Medical Education in Northeast China before Liberation[J].Chinese Journal of Medical History,1981,11(2):71

[15]Yu Dawang. Pharmaceutical Affair in China[J].Medicine and Pharmacology.1947,(2):9 ; (3):9

[16]Drug Training Weekly[J]. 4th, 11th, 18th October; 12th July, 20th June, 18th, 27th May

[17]Xue Yu. Make Omissions of Chinese Pharmacologic Education History[J]. Chinese Pharmaceutical Bulletin.1986,21(10):630

[18]Zhao Wenlin. The Fragment of Pharmacology History before Liberate[J]. Chinese Pharmaceutical Bulletin.1986,21(3):179

DOI 10.22448/AMJ.2017.3.22-23

THE MECHANISM RESEARCH ON ANTAGONIZING INFLAMMATORY INJURY BY ACUPUNCTURE BAI-HUI THROUGH QIU-BININ RATS WITH CEREBRAL HEMORRHAGE

\section{ChenQiu-xin}

The First Affiliated Hospital of Heilongjiang University Of ChineseMedicine,Haerbin, E-mail:chenqiuxin0451@126.com

Abstract Intracerebral hemorrhage $(\mathrm{ICH})$ is a kind of cerebrovascular disease with acute onset, severe illness, high mortality and high disability rate. How to effectively reduce the damage of nerve function has become

Амурский медицинский журнал №3 (19) 2017 
the focus of treatment of acute cerebral hemorrhage. At present, there are obvious inflammatory reactions in the brain hematoma and the surrounding tissues after intracerebral hemorrhage .In recent years, immunological studies have found that Toll like receptor 4 (TLR 4) biological function is the most prominent inflammatory reactions, TLR/ mediated nuclear factor (NF- K B) signaling pathway leads to brain injury and neurological deficit aggravate .In this experiment, through the establishment of rat autologous blood injection model of cerebral hemorrhage, observation of Baihui to Qubin effect on brain tissue in TLR - 4, TNF - alpha and IL 6 expression in rats and to explore the mechanism of hemorrhage in inflammatory injury in rats with cerebral hemorrhage.

Key words: acupuncture ,cerebral hemorrhage, tumor necrosis factor-a,Toll like receptor 4

The pathogenesis of cerebral hemorrhage is not yet clear, but related to atherosclerosis, inflammatory reaction, lipid metabolism disorders, abnormal coagulation mechanism and other factors. Cell immune inflammatory effects in the central nervous system, activation in normal pathological response in the central nervous system helps to restore homeostasis, but excessive activation is the release of a large number of inflammatory cytokines and cytotoxic substances, has become an important cause of neuronal death

Objective To observe the effect of penetrative needling of "Baihui" (GV 20) to "Qubin"(GB 7) on neurologic functions and expression og tumor necrosis factor (TNF-a) interleukin (IL-6) and toll-like receptor 4(TLR-4) in the tissue around the local cerebral hematoma in rats with intracerebral hemorrhage $(\mathrm{ICH})$, so as to provide evidence for clinical treatment of ICH.

Materials and methods 54 Wistar rats were randomly divided into sham operation group, model group and acupuncture group. Each group was divided into 3 subgroups according to the model of 1, 3 and $7 \mathrm{~d}$ after the model was established, and each group was divided into 6 groups. The rat model of intracerebral hemorrhage was established by autologous blood injection. The acupuncture group were "Baihui" to "Qubin" treatment, 24h treatment 1 times. Using the Longa score and body symmetry experiment score of neurological function after intracerebral hemorrhage rats were assessed by immunohistochemical hemorrhagic tissue Toll like receptor 4 was detected in brain (TLR 4), tumor necrosis factor alpha (TNF alpha), interleukin (IL - 6) positive expression.

Results and discussion Neurobehavioral:Model rats have limb paralysis,the acupuncture group compared with the model group, the neurological deficits were significantly reduced, the difference was significant $((P<0.01)$. Immunohistochemical results: In the sham group shows a small amount of brain tissue TNF- $\alpha$, IL- 6 and TLR4 protein expression at each time point;The expression of TNF- $\alpha$, IL-6 and TLR4 in the model group was higher than that in sham group $((\mathrm{P}<0.01)$.At each time point, the positive expression of TNF- $\alpha$, IL-6 and TLR4 in the acupuncture group was significantly lower than that in the model group ( $(\mathrm{P}<0.01)$.Correlation analysis of TLR-4 and IL-6 expression was positively correlated, and the correlation coefficient was 0.871, TLR4 and TNF alpha expression was positively correlated, and the correlation coefficient was 0.982.Conclusion:Acupuncture Bai-hui through Qiu-bin can inhibit the expression of TLR-4 and reduce the content of organization of hematoma of TNF- $\alpha$ and IL-6, reduce the inflammatory injury and improve the neurological function defect performance after cerebral hemorrhage.

Through the study of rat IL - 6, TNF - alpha and TLR 4 intracerebral hemorrhage, TLR 4 can induce inflammatory reaction in rats with intracerebral hemorrhage, and TLR - 4 and IL - 6 and TNF alpha expression was positively related to signal transduction pathway that mediated by TLR 4 final the IL - 6, TNF - alpha production, they are involved in the inflammatory response of cerebral hemorrhage, and acupuncture at Baihui Qubin can inhibit the expression of TLR 4, reduce the inflammatory factor IL - 6, TNF - alpha release, reduce inflammation, relieve nerve of rats injury, play a role in brain protection.

\section{References:}

[1]Liu XW,Zhou J,Peng P,et al.Study of acupuncture treatment timing on inflammation of experimental cerebral hemorrhage rats[J].Liaoning Journal of traditional chinese medicine,2014,41(6):1123-1125.

[2]Akira S,Uematsu S,Talceuchi O.Pathogen recognition and innate inmmunity[J].Cell,2006,124(4):783-801.

[3]HickenbottomSL,Grotta JC,Strong R,et al.Nuclear Factor- kB and Cell Death After Experimental Intracerebral Hemorrhage in Rats[J].Stroke, 1999,30:2472-2478.

[4]Kim MK,Choi TY,Lee MS, et al.Contralateral acupuncture versus ipsilateral acupuncture in the rehabilitation of post-stroke hemiplegic patients: a systematic review[J].BMC Complement Altern Med.2010,10:41.

\section{UDC: 579.61 DOI 10.22448/AMJ.2017.3.23-25 ADHESIVE ACTIVITY AND BIOFILM FORMING BY CULTURE OF ESCHERICHIA COLI}

\section{G.I. Chubenko, A.V. Prokopenko}

\section{Amur state medical academy. Blagovesshensk, Russia}

Abstracts. It is known that adhesion is the starting point of development of infectious process. Specific adhesion is the result of molecular interactions between the receptor cells and microbial adhesion. At least $65 \%$ of hospital infections are related to biofilms [1]. And bacteria of the family Enterobacteriaceae, together, are the most common causative agents of nosocomial infections [6]. In this connection, the studied adhesive activity of wild strains of E.coli, the formation of biofilms on a plastic surface with the use of an antioxidant of the flavonoid dihydroquercetin and without it.The study found a direct dependence of the rate of formation of biofilm and adhesion activity of microorganisms. 